RAIRO Operations Research

RAIRO Oper. Res. 36 (2002) 351-364

DOI: $10.1051 /$ ro:2003006

\title{
AN ALGORITHM FOR SOLVING MULTIPLE OBJECTIVE INTEGER LINEAR PROGRAMMING PROBLEM
}

\author{
Moncef Abbas $^{1}$ and Djamal ChaAbane ${ }^{1}$ \\ Communicated by Jean Abadie
}

\begin{abstract}
In the present paper a complete procedure for solving Multiple Objective Integer Linear Programming Problems is presented. The algorithm can be regarded as a corrected form and an alternative to the method that was proposed by Gupta and Malhotra. A numerical illustration is given to show that this latter can miss some efficient solutions. Whereas, the algorithm stated bellow determines all efficient solutions without missing any one.
\end{abstract}

Keywords. Multiple objective programming, integer linear programming.

\section{INTRODUCTION}

Multiple Integer Linear Programming problems have diverse applications such as agricultural planning, financial analysis of a firm, travelling salesman problems, Markovian replacement problems, the cutting stock problems, and portfolio selection problems.

It has been studied by several authors (e.g. Gupta and Malhotra [10], Klein [13], Bitran [7], Zionts [22], Abbas and Moulaï [1], etc.).

The main focus of this paper, is to find all the efficient points using the cutting plane technique and simple pivoting procedures. In the following section some theoretical tools are presented to prove the convergence of the proposed method followed by the algorithm, then lasted by a numerical illustration, conclusions and comments with short comparative indications to Gupta and Malhotra algorithm.

Received January, 2001.

1 Faculté de Mathématiques, Département de Recherche Opérationnelle, BP. 32, El-Alia Bab-Ezzouar, Alger, Algérie; e-mail: chaabane_dj@yahoo.fr

(c) EDP Sciences 2003 


\section{Theoretical TOOLS}

The multiple objective integer linear programming problem (MOILP) can be formulated as follows

$$
(P) \begin{cases}\operatorname{Max} Z_{1} & =C^{1} X \\ \operatorname{Max} Z_{2} & =C^{2} X \\ & \cdots \\ \operatorname{Max} Z_{p} & =C^{p} X \\ \text { subject to: } & X \in F=\left\{X \in \mathbb{R}^{n} / A X=b, X \text { positive integer }\right\}\end{cases}
$$

$A \in \mathbb{R}^{m \times n} ; b^{\prime} \in \mathbb{R}^{m} ; C^{i} \in \mathbb{R}^{n}$ for all $i \in I^{\prime}=\{1,2, \ldots, p\}$.

Definition 2.1. A point $X^{0} \in F$ is an efficient solution if there is no $X \in F$ such that : $Z_{i}(X) \geq Z_{i}\left(X^{0}\right)$ for all $i \in I^{\prime}$ and $Z_{i}(X)>Z_{i}\left(X^{0}\right)$ for at least one $i \in I^{\prime}$. Otherwise, $X^{0}$ is not efficient and $\left(Z_{1}\left(X^{0}\right), Z_{2}\left(X^{0}\right), \ldots, Z_{p}\left(X^{0}\right)\right)$ is said to be a dominated p-tuple.

The problem $(P 1)$ is defined as follows:

$$
(P 1) \begin{cases}\operatorname{Max} Z_{1} & =C^{1} X \\ \text { subject to: } & X \in F .\end{cases}
$$

The relaxed problem is:

$$
\begin{aligned}
& \left(P_{R}\right) \begin{cases}\operatorname{Max} Z_{1} & =C^{1} X \\
\text { subject to: } & X \in F .\end{cases} \\
& F_{R}=\left\{X \in \mathbb{R}^{n} / A X=b, X \text { is positive }\right\} .
\end{aligned}
$$

Definition 2.2. $\quad$ An optimal solution $X^{0}$ of the problem $(P 1)$ is said to be unique if there does not exist $X^{1} \in F$ such that $X^{1} \neq X^{0}$ with $Z_{1}\left(X^{0}\right)=Z_{1}\left(X^{1}\right)$. Otherwise, the problem $(P 1)$ has got at least one alternate solution over he region $F$.

\section{Notations}

- $X_{1}^{*}$ : is the optimal solution of the problem $(P 1)$. This is obtained by applying Gomory cuts over the problem $\left(P_{R}\right)$.

- $Z_{1}^{1}=Z_{1}^{*}$ : is the optimal solution obtained in $(P 1)$.

- $\left(Z_{1}^{1}, Z_{2}^{1}, \ldots, Z_{p}^{1}\right)$ : is the first non-dominated $p$-tuple, where: $Z_{i}^{1}=Z_{i}\left(X_{1}^{*}\right)$ for $i=2,3, \ldots, p$.

For $k \geq 1$ :

- $X_{k}$ : is the efficient solution underlying $\left(Z_{1}^{k}, Z_{2}^{k}, \ldots, Z_{p}^{k}\right)$;

- $B_{k}$ : basis associated with $X_{k}$;

- $a_{k j}$ : activity vector of $x_{k j}$ with respect to the truncated region;

- $y_{k j}=\left(B_{k}\right)^{-1} a_{k j}$;

- $I_{k}=\left\{j / a_{k j} \in B_{k}\right\}$;

- $N_{k}=\left\{j / a_{k j} \notin B_{k}\right\}$; 
- $\Gamma_{k}=\left\{\begin{array}{c}j \in N_{k} / z_{1 j}-c_{j}^{1}>0 \text { and } z_{i j}-c_{j}^{i}>0 \text { for at } \\ \text { least one } i \in\{2,3, \ldots, p\}\end{array}\right\} ;$
- $E_{j_{k}}=\left\{\begin{array}{c}x_{i}=x_{k i}-\theta_{j_{k}} y_{k, i j_{k}} \\ \left(x_{i}\right) \in \mathbb{R}^{n} / \quad \begin{array}{c}x_{j_{k}}=\theta_{j_{k}} \\ x_{\alpha}=0\end{array} \text { for all } \alpha \in N_{k} \backslash\left\{j_{k}\right\}\end{array}\right\}$.

Where $\theta_{j_{k}}$ is the integer value between 0 and $\min _{i \in I_{k}}\left\{\frac{x_{k i}}{y_{k, i j_{k}}} ; y_{k, i j_{k}}>0\right\}$ and $\theta_{j_{k}}, \theta_{j_{k}} \times y_{k, i j_{k}}$ are integers.

Theorem 2.1. All integer feasible solutions of problem $(P 1)$ alternate to $X_{1}$ on edge $E_{j_{1}}, j_{1} \in \Gamma_{1}$ in the region $F$ (or truncated region of $F$ ) emanating from it lie in the open half space $\sum_{j \in N_{1} \backslash\left\{j_{1}\right\}} x_{j}<1$.

Proof. We have $A X_{1}=b\left(X_{1}\right.$ is a feasible solution of $\left.(P 1)\right) . A X_{1}=\sum_{i \in I_{1}} a_{1 i} x_{1 i}=b$. For some $j_{1} \in \Gamma_{1}, \sum_{i \in I_{1}} a_{1 i} x_{1 i}-\theta_{j_{1}} a_{1 j_{1}}+\theta_{j_{1}} a_{1 j_{1}}=b$, where $\theta_{j_{1}}$ is a nonzero positive scalar. $\sum_{i \in I_{1}} a_{1 i} x_{1 i}-\theta_{j_{1}}\left(\sum_{i \in I_{1}} a_{1 i} y_{1}, i j_{1}\right)+\theta_{j_{1}} a_{1 j_{1}}=b ; \sum_{i \in I_{1}} a_{1 i}\left(x_{1 i}-\theta_{j_{1}} y_{1}, i j_{1}\right)+$ $\theta_{j_{1}} a_{1 j_{1}}=b$.

For $0 \leq \theta_{j_{1}} \leq \min _{i \in I_{1}}\left\{\frac{x_{1 i}}{y_{1, i j_{1}}} ; y_{1, i j_{1}}>0\right\}, X_{2}$ is defined as follows:

$$
X_{2}=\left\{\begin{array}{l}
x_{2 i}=x_{1 i}-\theta_{j_{1}} y_{1}, i j_{1} i \in I_{1} \\
x_{2 j_{1}}=\theta_{j_{1}} \\
x_{2 \alpha}=0 \text { for all } \alpha \in N_{1} \backslash\left\{j_{1}\right\}
\end{array}\right\} ; \text { which is a new }
$$

integer feasible solution of $(P 1)$ provided $\theta_{j_{1}}$ and $\theta_{j_{1}} \times y_{1, i j_{1}}$ are positive integers.

We show now that $Z_{1}\left(X_{2}\right)=Z_{1}\left(X_{1}\right)$.

$$
\begin{aligned}
Z_{1}\left(X_{2}\right) & =\sum_{i \in I_{2}} c_{1 i} x_{2 i}=\sum_{i \in I_{1}}\left(x_{1 i}-\theta_{j_{1}} y_{1, i j_{1}}\right) c_{1 i}+c_{1 j_{1}} \theta_{j_{1}} \\
& =\sum_{i \in I_{1}} c_{1 i} x_{1 i}-\sum_{i \in I_{1}} c_{1 i} \theta_{j_{1}} y_{1}, i j_{1}+c_{1 j_{1}} \theta_{j_{1}} \\
& =\sum_{i \in I_{1}} c_{1 i} x_{1 i}-\theta_{j_{1}} \sum_{i \in I_{1}} c_{1 i} y_{1}, i j_{1}+c_{1 j_{1}} \theta_{j_{1}} \\
& =\sum_{i \in I_{1}} c_{1 i} x_{1 i}-\theta_{j_{1}}\left(\sum_{i \in I_{1}} c_{1 i} y_{1, i j_{1}}-c_{1 j_{1}}\right) \\
& =\sum_{i \in I_{1}} c_{1 i} x_{1 i}=Z_{1}\left(X_{1}\right)
\end{aligned}
$$

since $\sum_{i \in I_{1}} c_{1 i} y_{1}, i j_{1}-c_{1 j_{1}}=0$. 
Therefore, $X_{2}$ is an integer feasible solution of $\left(P_{1}\right)$, alternate to $X_{1}$ lying on an edge

$$
E_{j_{1}}=\left\{\begin{aligned}
& x_{2 i}=x_{1 i}-\theta_{j_{1}} y_{1}, i j_{1} \\
&\left(x_{i}\right) \in \mathbb{R}^{\left(\left|I_{1}\right|+\left|N_{1}\right|\right) /} / I_{1} \\
& x_{2 j_{1}}=\theta_{j_{1}} \\
& x_{2 \alpha}=0 \text { for all } \alpha \in N_{1} \backslash\left\{j_{1}\right\}
\end{aligned}\right\} .
$$

We have, $\sum_{j \in N_{1} \backslash\left\{j_{1}\right\}} x_{2 j}<1$; since $x_{2 j}=0$ for all $j \in N_{1} \backslash\left\{j_{1}\right\}$. Thus, the point $X_{2}$ lies in the open half space $\sum_{j \in N_{1} \backslash\left\{j_{1}\right\}} x_{j}<1$.

Corollary 2.1. An integer feasible solution of problem $(P 1)$ not on edge $E_{j_{1}}$, $j_{1} \in \Gamma_{1}$ in the truncated region $F$ throughout the best solution $X_{1}$ of $(P 1)$ lies in $\sum_{j \in N_{1} \backslash\left\{j_{1}\right\}} x_{j} \geq 1$.

Proof. Let $\tilde{X}=\left(\widetilde{x}_{j}\right)_{i \in I_{1} \cup N_{1}}$ be an integer feasible solution of problem $(P 1)$ not on an edge $E_{j_{1}}$ such that: $\sum_{j \in N_{1} \backslash\left\{j_{1}\right\}} \widetilde{x}_{j}<1$. Then $\widetilde{x}_{j}=0$ for all $j \in N_{1} \backslash\left\{j_{1}\right\}$.

- $\widetilde{x}_{j_{1}}>\mathbf{0},\left(\tilde{X}\right.$ is alternate to $\left.X_{1}\right)$ then, $\tilde{X}$ must lie in the direction of vector $a_{1 j_{1}}, j_{1} \in \Gamma_{1}$.

* $\widetilde{x}_{j_{1}}>\min _{i \in I_{1}}\left\{\frac{x_{1 i}}{y_{1, i j_{1}}} ; y_{k, i j_{1}}>0\right\}=\frac{x_{1 q}}{y_{1, i j_{1}}}$ (for example), then $\widetilde{x}_{q}=$ $\widetilde{x}_{1 q}-\widetilde{x}_{j_{1}} y_{1, q j}<0$ which implies infeasibility of $\widetilde{X}_{1}$ contradicting to the hypothesis.

$* \widetilde{x}_{j_{1}}$ is a positive integer such that: $\widetilde{x}_{j_{1}} \leq \min _{i \in I_{1}}\left\{\frac{x_{1 i}}{y_{1, i j_{1}}} ; y_{k, i j_{1}}>0\right\}$, this also implies that $\widetilde{X}$ lies on the edge $E_{j_{1}}$, which is not true.

Hence, $\widetilde{x}_{j}>0$ for at least one $j \in N_{1} \backslash\left\{j_{1}\right\}$.

- $\widetilde{x}_{j_{1}}=\mathbf{0},\left(\widetilde{X}\right.$ is not alternate to $\left.X_{1}\right)$ then, the index set of non-basic variables in the optimal table corresponding to $\widetilde{X}$ is the same as $N_{1}$ since $N_{1}=N_{1} \backslash\left\{j_{1}\right\} \cup\left\{j_{1}\right\}$, therefore the index set of basic variables in the optimal table corresponding to $\widetilde{X}$ is the same as $B$. Thus $\widetilde{X}=X_{1}$ which is not the case.

Hence $\widetilde{x}_{j}>0$ for at least one $j \in N_{1} \backslash\left\{j_{1}\right\}$. It is shown now, that $\widetilde{x}_{j}>0$ for at least one $j \in N_{1} \backslash\left\{j_{1}\right\}$. Therefore, $\widetilde{X}$ lies in the closed half space $\sum_{j \in N_{1} \backslash\left\{j_{1}\right\}} x_{j} \geq 1$.

\section{THE ALGORITHM}

The purpose of the algorithm is to determine all efficient solutions of the problem $(P)$. Starting with an initial efficient solution derived from solving $(P 1)$, 
Dantzig cuts and Gomory cuts (if necessary) are used in sequence after having scanned the edges $E_{k}$. The output of the algorithm gives all efficient solutions existing in the bounded set $F$. without missing any one. This will be justified later.

Step 1. Solve the problem $(P 1)$. Instead of $(P 1)$ one can constructs one of the problems $(P i ; i=2,3, \ldots, p)$ and solve it.

- If the optimal solution is unique, then record the first non-dominated ptuples $\left(Z_{1}^{1}, Z_{2}^{1}, \ldots, Z_{p}^{1}\right)$ and put it in the set of non-dominated solutions $\mathbf{O p t}_{0}$. Go to Step 2.

- If the optimal solution of the problem $(P 1)$ is not unique.

Find all alternate solutions to $X_{1}^{*}$; for each solution evaluate the criterions and eliminate all dominated p-tuples to obtain $\mathbf{O p t}_{0}$. Consider the p-tuple $\left(Z_{1}, Z_{2}\right.$, $\left.\ldots Z_{p}\right)$ as the first non-dominated p-tuple such that $Z_{2}$ is the best among all second criterions in $\mathbf{O p t}_{0}$. If there exists a tie, the p-tuple considered will have the best value of the third criterion with respect to all p-tuples in $\mathbf{O p t}_{0}$ and the process continue whenever a tie appears. Rename the first efficient p-tuple found as $\left(Z_{1}^{1}, Z_{2}^{1}, \ldots Z_{p}^{1}\right)$. Then, go to Step 2 .

Step 2. Let $k=1$.

2.1. Construct the set $\Gamma_{k}$.

2.1.1. If $\Gamma_{k}=\emptyset$ then, choose any $j_{k} \in N_{k}$ and go to (2.2).

2.1.2. Else, choose any $j_{k} \in \Gamma_{k}$. Go to (2.2).

2.2. Calculate $\theta_{k j_{k}}=\operatorname{Min}_{i \in I_{k}}\left\{\frac{x_{k i}}{y_{k}, i j_{k}} ; y_{k, i j_{k}}>0\right\}$.

Let $\theta_{k j_{k}}^{0}$ be the integer part of $\theta_{k j_{k}}$.

- If $\theta_{k j_{k}}^{0}<1$ then ignore it and go to (2.3).

- If not, for $\theta=1,2, \ldots, \theta_{k j_{k}}^{0}$; calculate all integer feasible solutions lying on the edge $E_{j_{k}}$ using $\left\{\begin{array}{l}x_{i}=x_{k i}-\theta \times y_{k}, i_{j_{k}} \\ x_{j_{k}}=\theta \\ x_{\alpha}=0 \text { for all } \alpha \in N_{k} \backslash\left\{j_{k}\right\} .\end{array}\right.$

Evaluate all the criterions at each of these solutions and add only non-dominated criterions in the set $\mathbf{O} \mathbf{p} \mathbf{t}_{k-1}$ to obtain the new set Opt $_{k}$. Go to (2.3).

2.3. Truncate the solution by $\sum_{j \in N_{k} \backslash\left\{j_{k}\right\}} x_{j} \geq 1$.

Apply the dual simplex method to obtain positive feasible solution $X_{k+1}$. Evaluate all the criterions at this solution and add the p-tuple corresponding into $\mathbf{O} \mathbf{p t}_{k}$ to obtain the new set $\mathbf{O} \mathbf{p t} \mathbf{t}_{k+1}$ if it is not dominated, else ignore it. Go to Step. 3.

Step 3. If $X_{k+1}$ obtained in (2.3) is integer positive infeasible solution (lying outside $F$ ) then stop. Otherwise put $k=k+1$ and return to (2.1).

Proposition 1. The algorithm above converges in a finite number of steps. 


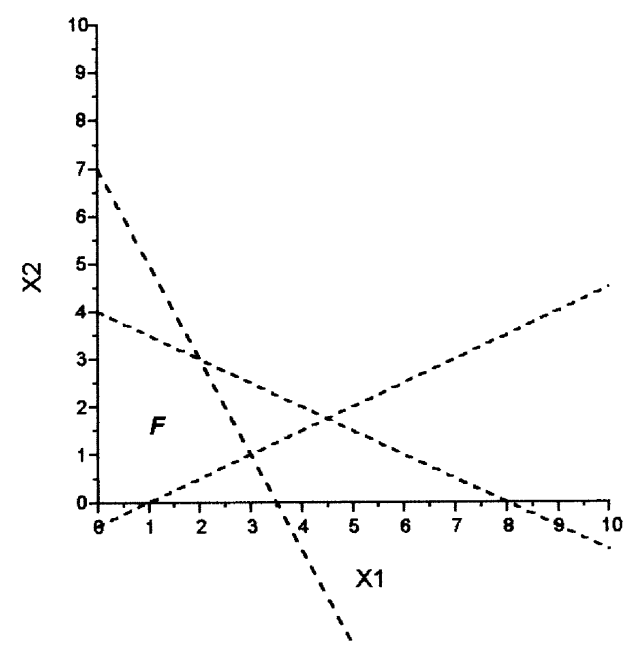

Figure 1.

Proof. The feasible region $F$ is being truncated at each step by respected application of the cuts $\sum_{j \in N_{k} \backslash\left\{j_{k}\right\}} x_{j} \geq 1$ and Gomory cut is used whenever needed. The entire region $F$ is scanned such that, an edge or a point once deleted can not reappear again. Therefore, the algorithm converges in a finite number of steps.

\section{Numerical ILlUStration}

$\operatorname{Max} Z_{1}=x_{1}-3 x_{2}$

$\operatorname{Max} Z_{2}=x_{1}+3 x_{2}$

Subject to

Consider the following example

$$
\begin{aligned}
& x_{1}+2 x_{2} \leq 8 \\
& 2 x_{1}+x_{2} \leq 7 \\
& x_{1}-2 x_{2} \leq 1 \\
& x_{1}, x_{2} \geq 0 \text { and integers }
\end{aligned}
$$

The region $F$ is shown in the figure above.

First $(P 1)$ is being solved. 
TABLE 1.

\begin{tabular}{lccrrrrr}
\hline \hline Basis & $\begin{array}{c}\text { value of } \\
\text { basic } \\
\text { variable }\end{array}$ & $x_{1}$ & $x_{2}$ & $x_{3}$ & $x_{4}$ & $x_{5}$ \\
\hline$x_{3}$ & 7 & 0 & 4 & 1 & 0 & -1 \\
$x_{4}$ & 5 & 0 & 5 & 0 & 1 & -2 \\
$x_{1}$ & 1 & 1 & -2 & 0 & 0 & 1 \\
\hline \hline$Z_{j}^{C^{1}}-C_{j}^{1}$ & 1 & 0 & 1 & 0 & 0 & 1 \\
$Z_{j}^{C^{2}}-C_{j}^{2}$ & 1 & 0 & -5 & 0 & 0 & 1 \\
\hline \hline
\end{tabular}

The solution is unique and it is given in the table above: $X_{1}=X_{1}^{*}=(1,0)$ and the first non-dominated couple $\left(Z_{1}^{1}, Z_{2}^{1}\right)=(1,1)$.

$k=1$,

$$
\mathbf{O p t}_{0}=\left\{\left(\begin{array}{l}
1 \\
1
\end{array}\right)\right\} \text {. }
$$

We have: $I_{1}=\{1,3,4\}, N_{1}=\{2,5\}$.

$\Gamma_{1}=\left\{\begin{array}{c}j \in N_{1} / z_{1 j}-c_{j}^{1}>0 \text { and } z_{i j}-c_{j}^{i}>0 \text { for at } \\ \text { least one } i \in\{2\}\end{array}\right\}=\{5\}$.

$j_{1}=5 ; \theta_{15}=\operatorname{Min}\left\{\frac{1}{1}\right\}=1 ; \theta_{15}^{0}=1$.

The solution on the edge $E_{5}$ is: $\left\{\begin{array}{l}x_{3}^{1}=7-(-1)=8 \\ x_{4}^{1}=5-(-2)=7 \\ x_{1}^{1}=1-(1)=0 \\ x_{5}^{1}=1 \\ x_{2}^{1}=0 .\end{array}\right.$

Thus, $Z^{1,1}=\left(\begin{array}{l}0 \\ 0\end{array}\right)$ which is dominated.

Truncate by $x_{2} \geq 1$ (see Fig. 2 bellow) and $-x_{2}+x_{6}=-1$ is to be added at the bottom of the previous table.

TABLE 2 .

\begin{tabular}{lccrrrrrr}
\hline \hline Basis & $\begin{array}{c}\text { value of } \\
\text { basic } \\
\text { variable }\end{array}$ & $x_{1}$ & $x_{2}$ & $x_{3}$ & $x_{4}$ & $x_{5}$ & $x_{6}$ \\
\hline$x_{3}$ & 7 & 0 & 4 & 1 & 0 & -1 & 0 \\
$x_{4}$ & 5 & 0 & 5 & 0 & 1 & -2 & 0 \\
$x_{1}$ & 1 & 1 & -2 & 0 & 0 & 1 & 0 \\
$x_{6}$ & -1 & 0 & -1 & 0 & 0 & 0 & 1 \\
\hline \hline$Z_{j}^{C^{1}}-C_{j}^{1}$ & 1 & 0 & 1 & 0 & 0 & 1 & 0 \\
$Z_{j}^{C^{2}}-C_{j}^{2}$ & 1 & 0 & -5 & 0 & 0 & 1 & 0 \\
\hline \hline
\end{tabular}




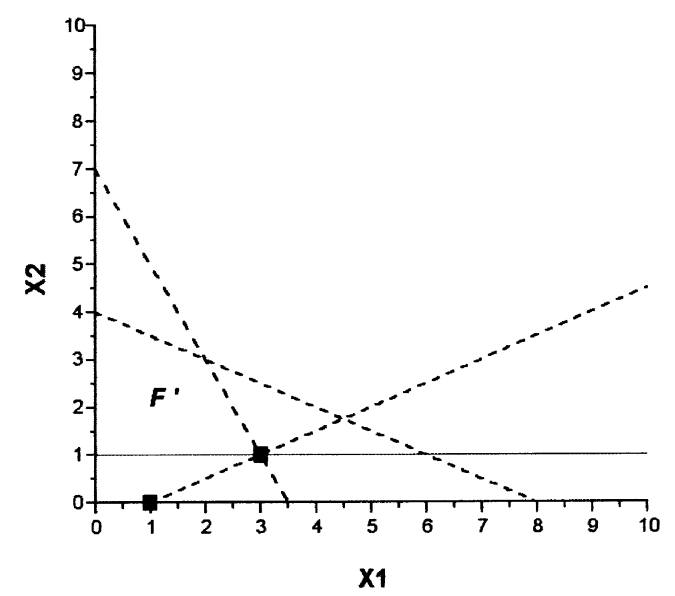

FiguRE 2.

Applying the dual simplex method we obtain:

TABLE 3 .

\begin{tabular}{lcccccccr}
\hline \hline Basis & $\begin{array}{c}\text { value of } \\
\text { basic } \\
\text { variable }\end{array}$ & $x_{1}$ & $x_{2}$ & $x_{3}$ & $x_{4}$ & $x_{5}$ & $x_{6}$ \\
\hline$x_{3}$ & 3 & 0 & 0 & 1 & 0 & -1 & 4 \\
$x_{4}$ & 0 & 0 & 0 & 0 & 1 & -2 & 5 \\
$x_{1}$ & 3 & 1 & 0 & 0 & 0 & 1 & -2 \\
$x_{2}$ & 1 & 0 & 1 & 0 & 0 & 0 & -1 \\
\hline \hline$Z_{j}^{C^{1}}-C_{j}^{1}$ & 0 & 0 & 0 & 0 & 0 & 1 & 1 \\
$Z_{j}^{C^{2}}-C_{j}^{2}$ & 6 & 0 & 0 & 0 & 0 & 1 & -5 \\
\hline \hline
\end{tabular}

The solution is $X_{2}=(3,1)$ yields to $\left(Z_{1}^{2}, Z_{2}^{2}\right)=(0,6)$ which is non-dominated with regards to the previous.

Thus $\mathbf{O p t}_{2}=\mathbf{O p t}_{1} \cup\left\{\left(\begin{array}{l}0 \\ 6\end{array}\right)\right\}=\left\{\left(\begin{array}{l}1 \\ 1\end{array}\right),\left(\begin{array}{l}0 \\ 6\end{array}\right)\right\}$.

$k=2$;

$I_{2}=\{1,2,3,4\}, N_{2}=\{5,6\}$.

$\Gamma_{2}=\{5\}$ and $\theta_{25}^{0}=3$.

Calculate the solutions on the edge $E_{5}$

$\theta_{2}=1 ;\left\{x_{3}^{1}=4, x_{4}^{1}=2, x_{1}^{1}=2, x_{2}^{1}=1, x_{5}^{1}=1, x_{6}^{1}=0\right\}$

$\Rightarrow Z^{2,1}=\left(\begin{array}{c}-1 \\ 5\end{array}\right)$ which is dominated.

$\theta_{2}=2 ;\left\{x_{3}^{2}=5, x_{4}^{2}=4, x_{1}^{2}=1, x_{2}^{2}=1, x_{5}^{2}=1, x_{6}^{2}=0\right\}$

$\Rightarrow Z^{2,2}=\left(\begin{array}{c}-2 \\ 4\end{array}\right)$ which is dominated. 


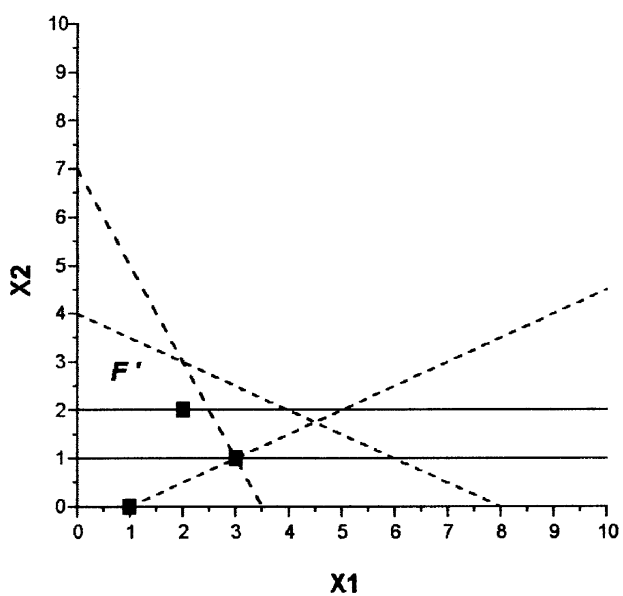

FiguRE 3.

$\theta_{2}=3 ;\left\{x_{3}^{3}=6, x_{4}^{3}=6, x_{1}^{3}=0, x_{2}^{3}=1, x_{5}^{3}=1, x_{6}^{3}=0\right\}$

$\Rightarrow Z^{2,3}=\left(\begin{array}{c}-3 \\ 3\end{array}\right)$ which is dominated.

The edge $E_{5}$ is dominated for all $j \in \Gamma_{2}$. Thus, according to Gupta and Malhotra the procedure is terminated. But, the remaining efficient solutions are not yet detected.

Within the algorithm proposed, the cut $\sum_{j \in N_{2} \backslash\{5\}} x_{j} \geq 1$ is being performed. Then, $x_{6} \geq 1$ (equivalently $x_{2} \geq 2$ - see Fig. 3 ) is added to the previous tableau and Gomory cuts and dual simplex method are used to obtain the following table:

TABLE 4 .

\begin{tabular}{lccccccccrr}
\hline \hline Basis & $\begin{array}{c}\text { value of } \\
\text { basic } \\
\text { variable }\end{array}$ & $x_{1}$ & $x_{2}$ & $x_{3}$ & $x_{4}$ & $x_{5}$ & $x_{6}$ & $x_{7}$ & $x_{8}$ \\
& 2 & 0 & 0 & 1 & 0 & 0 & 0 & 2 & $-1 / 2$ \\
\hline$x_{3}$ & 3 & 0 & 0 & 0 & 0 & 1 & 0 & -2 & $-1 / 2$ \\
$x_{5}$ & 2 & 1 & 0 & 0 & 0 & 0 & 0 & 0 & $1 / 2$ \\
$x_{1}$ & 2 & 0 & 1 & 0 & 0 & 0 & 0 & -1 & 0 \\
$x_{2}$ & 1 & 0 & 0 & 0 & 0 & 0 & 1 & -1 & 0 \\
$x_{6}$ & 1 & 0 & 0 & 0 & 1 & 0 & 0 & 1 & -1 \\
$x_{4}$ & -4 & 0 & 0 & 0 & 0 & 0 & 0 & 3 & $1 / 2$ \\
\hline \hline$Z_{j}^{C^{1}}-C_{j}^{1}$ & 8 & 0 & 0 & 0 & 0 & 0 & 0 & -3 & $1 / 2$ \\
$Z_{j}^{C^{2}}-C_{j}^{2}$ & 8
\end{tabular}


The solution $X_{3}=(2,2)$ yields non dominated couple $\left(Z_{1}^{3}, Z_{2}^{3}\right)=(-4,8)$. $\mathbf{O p t}_{3}=\left\{\left(\begin{array}{l}1 \\ 1\end{array}\right),\left(\begin{array}{l}0 \\ 6\end{array}\right),\left(\begin{array}{c}-4 \\ 8\end{array}\right)\right\}$.

$k=3$;

$I_{3}=\{1,2,3,4,5,6\} ; N_{3}=\{7,8\}$.

$\Gamma_{3}=\{8\} \Rightarrow \theta_{38}^{0}=4$.

$\theta_{3}=1$; the solution is infeasible.

$\theta_{3}=2 ;\left\{\begin{array}{c}x_{3}^{3}=3, x_{5}^{3}=4, x_{1}^{3}=1, x_{2}^{3}=2, x_{6}^{3}=1, x_{4}^{3}=3, \\ x_{8}^{3}=2, x_{7}^{3}=0\end{array}\right\}$

yielding to $Z^{3,1}=\left(\begin{array}{c}-5 \\ 7\end{array}\right)$ dominated.

$\theta_{3}=3$; the solution is infeasible.

$\theta_{3}=4 ;\left\{\begin{array}{c}x_{3}^{3}=4, x_{5}^{3}=5, x_{1}^{3}=0, x_{2}^{3}=2, x_{6}^{3}=1, x_{4}^{3}=5, \\ x_{8}^{3}=4, x_{7}^{3}=0\end{array}\right\}$

yielding to $Z^{3,2}=\left(\begin{array}{c}-6 \\ 6\end{array}\right)$ which is dominated.

Add the cut $x_{7} \geq 1\left(x_{2} \geq 3\right.$ as shown in Fig. 4) and apply the dual simplex to obtain the following results.

\section{TABle 5.}

\begin{tabular}{lcccccccccrr}
\hline \hline Basis & $\begin{array}{c}\text { value of } \\
\text { basic } \\
\text { variable }\end{array}$ & $x_{1}$ & $x_{2}$ & $x_{3}$ & $x_{4}$ & $x_{5}$ & $x_{6}$ & $x_{7}$ & $x_{8}$ & $x_{9}$ \\
& 0 & 0 & 0 & 1 & 0 & 0 & 0 & 0 & $1 / 2$ & 2 \\
\hline$x_{3}$ & 5 & 0 & 0 & 0 & 0 & 1 & 0 & 0 & $-1 / 2$ & -2 \\
$x_{5}$ & 2 & 1 & 0 & 0 & 0 & 0 & 0 & 0 & $1 / 2$ & 0 \\
$x_{1}$ & 3 & 0 & 1 & 0 & 0 & 0 & 0 & 0 & 0 & -1 \\
$x_{2}$ & 2 & 0 & 0 & 0 & 0 & 0 & 1 & 0 & 0 & -1 \\
$x_{6}$ & 0 & 0 & 0 & 0 & 1 & 0 & 0 & 0 & -1 & 1 \\
$x_{4}$ & 1 & 0 & 0 & 0 & 0 & 0 & 0 & 1 & 0 & -1 \\
$x_{7}$ & -7 & 0 & 0 & 0 & 0 & 0 & 0 & 0 & $1 / 2$ & 3 \\
\hline \hline$Z_{j}^{C^{1}}-C_{j}^{1}$ & 11 & 0 & 0 & 0 & 0 & 0 & 0 & 0 & $1 / 2$ & -3 \\
$Z_{j}^{C^{2}}-C_{j}^{2}$ & 11 & & & & & & & & & & \\
\hline \hline
\end{tabular}

The solution $X_{4}=(2,3)$ yields to $\left(Z_{1}^{4}, Z_{2}^{4}\right)=(-7,11)$ which is non-dominated.

$k=4$;

$\mathbf{O p t}_{4}=\left\{\left(\begin{array}{l}1 \\ 1\end{array}\right),\left(\begin{array}{l}0 \\ 6\end{array}\right),\left(\begin{array}{c}-4 \\ 8\end{array}\right),\left(\begin{array}{c}-7 \\ 11\end{array}\right)\right\}$.

$I_{4}=\{1,2,3,4,5,6,7\} ; N_{4}=\{8,9\}$.

$\Gamma_{4}=\{8\} \Rightarrow \theta_{48}^{0}=4$.

$\theta_{4}=1$; the solution is infeasible. 


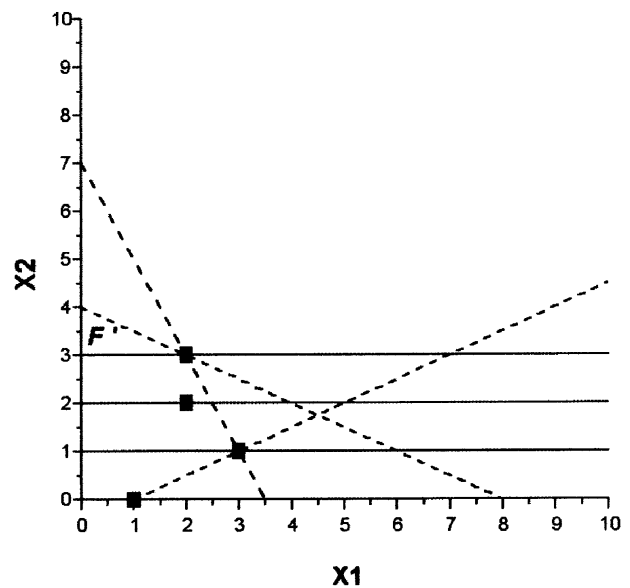

Figure 4.

$\theta_{4}=2 ;\left\{\begin{array}{c}x_{3}^{4}=1, x_{5}^{4}=6, x_{1}^{4}=1, x_{2}^{4}=3, x_{6}^{4}=2, x_{4}^{4}=2, \\ x_{7}^{4}=1, x_{8}^{4}=2, x_{9}^{4}=0\end{array}\right\}$ yielding to $Z^{4,1}=\left(\begin{array}{c}-8 \\ 11\end{array}\right)$ dominated.

$\theta_{4}=3$; the solution is infeasible.

$\theta_{4}=4 ;\left\{\begin{array}{c}x_{3}^{4}=2, x_{5}^{4}=7, x_{1}^{4}=0, x_{2}^{4}=3, x_{6}^{4}=2, x_{4}^{4}=4, \\ x_{7}^{4}=1, x_{8}^{4}=4, x_{9}^{4}=0\end{array}\right\}$

yielding to $Z^{4,2}=\left(\begin{array}{c}-9 \\ 9\end{array}\right)$ which is dominated.

Add the cut $x_{9} \geq 1\left(x_{2} \geq 4\right.$ as shown in Fig. 5$)$ to the Table 5 and apply the dual simplex method.

TABLE 6 .

\begin{tabular}{lccccccccccr}
\hline \hline Basis & $\begin{array}{c}\text { value of } \\
\text { basic } \\
\text { variable }\end{array}$ & $x_{1}$ & $x_{2}$ & $x_{3}$ & $x_{4}$ & $x_{5}$ & $x_{6}$ & $x_{7}$ & $x_{8}$ & $x_{9}$ & $x_{10}$ \\
& 4 & 0 & 0 & -2 & 0 & 0 & 0 & 0 & 1 & 0 & 0 \\
\hline$x_{8}$ & 9 & 0 & 0 & -1 & 0 & 1 & 0 & 0 & 0 & 0 & -2 \\
$x_{5}$ & 0 & 1 & 0 & 1 & 0 & 0 & 0 & 0 & 0 & 0 & 0 \\
$x_{1}$ & 4 & 0 & 1 & 0 & 0 & 0 & 0 & 0 & 0 & 0 & -1 \\
$x_{2}$ & 3 & 0 & 0 & 0 & 0 & 0 & 1 & 0 & 0 & 0 & -1 \\
$x_{6}$ & 1 & 0 & 0 & -2 & 1 & 0 & 0 & 0 & 0 & 0 & -1 \\
$x_{4}$ & 2 & 0 & 0 & 0 & 0 & 0 & 0 & 1 & 0 & 0 & -1 \\
$x_{7}$ & 1 & 0 & 0 & 0 & 0 & 0 & 0 & 0 & 0 & 1 & -1 \\
$x_{9}$ & -12 & 0 & 0 & 1 & 0 & 0 & 0 & 0 & 0 & 0 & 3 \\
\hline \hline$Z_{j}^{C^{1}}-C_{j}^{1}$ & 12 & 0 & 0 & 1 & 0 & 0 & 0 & 0 & 0 & 0 & -3 \\
$Z_{j}^{C^{2}}-C_{j}^{2}$ & 12 &
\end{tabular}




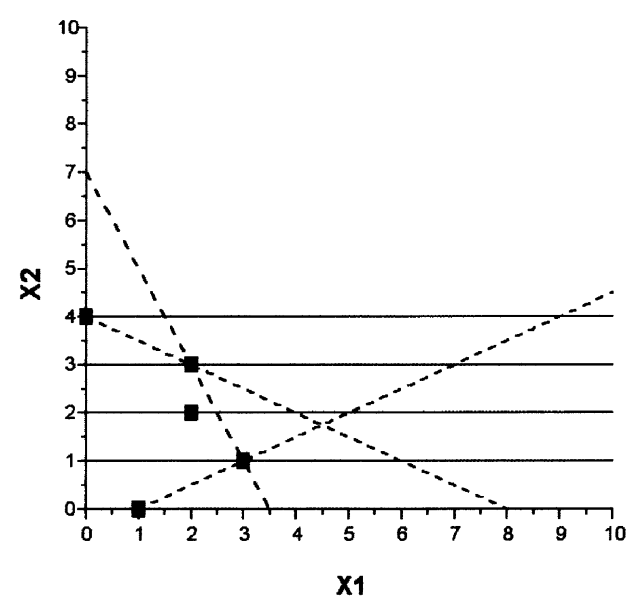

Figure 5.

The solution $X_{5}=(0,4)$ yields to $Z_{5}=\left(\begin{array}{c}-12 \\ 12\end{array}\right)$ which is non-dominated. $\mathbf{O p t}_{5}=\left\{\left(\begin{array}{l}1 \\ 1\end{array}\right),\left(\begin{array}{c}0 \\ 6\end{array}\right),\left(\begin{array}{c}-4 \\ 8\end{array}\right),\left(\begin{array}{c}-7 \\ 11\end{array}\right),\left(\begin{array}{c}-12 \\ 12\end{array}\right)\right\}$.

$\Gamma_{5}=\{3\} \Rightarrow \theta_{53}^{0}=0<1$. Ignore it.

Cut with $x_{10} \geq 1$ (equivalently $x_{2} \geq 5$ ) which lies outside the region $F$. If we apply one iteration of the dual simplex technique we achieve to an infeasible solution (see the table bellow) $X_{6}=(0,5) \notin F$.

The algorithm terminates with the set of all non-dominated $\mathbf{O p t}_{4}$ and the set of the efficient solutions (square dots in Fig. 5) is given by: $E f f=\{(1,0),(3,1)$, $(2,2),(2,3),(0,4)\}$.

TABLE 7.

\begin{tabular}{lcccccccccccr}
\hline \hline Basis & $\begin{array}{c}\text { value of } \\
\text { basic } \\
\text { variable }\end{array}$ & $x_{1}$ & $x_{2}$ & $x_{3}$ & $x_{4}$ & $x_{5}$ & $x_{6}$ & $x_{7}$ & $x_{8}$ & $x_{9}$ & $x_{10}$ & $x_{11}$ \\
& 4 & 0 & 0 & -2 & 0 & 0 & 0 & 0 & 1 & 0 & 0 & 0 \\
\hline$x_{8}$ & 11 & 0 & 0 & -1 & 0 & 1 & 0 & 0 & 0 & 0 & 0 & -2 \\
$x_{5}$ & 0 & 1 & 0 & 1 & 0 & 0 & 0 & 0 & 0 & 0 & 0 & 0 \\
$x_{1}$ & 5 & 0 & 1 & 0 & 0 & 0 & 0 & 0 & 0 & 0 & 0 & -1 \\
$x_{2}$ & 4 & 0 & 0 & 0 & 0 & 0 & 1 & 0 & 0 & 0 & 0 & -1 \\
$x_{6}$ & 2 & 0 & 0 & -2 & 1 & 0 & 0 & 0 & 0 & 0 & 0 & -1 \\
$x_{4}$ & 3 & 0 & 0 & 0 & 0 & 0 & 0 & 1 & 0 & 0 & 0 & -1 \\
$x_{7}$ & 2 & 0 & 0 & 0 & 0 & 0 & 0 & 0 & 0 & 1 & 0 & -1 \\
$x_{9}$ & 1 & 0 & 0 & 0 & 0 & 0 & 0 & 0 & 0 & 0 & 1 & -1 \\
$x_{10}$ & -15 & 0 & 0 & 1 & 0 & 0 & 0 & 0 & 0 & 0 & 0 & 3 \\
\hline \hline$Z_{j}^{C^{1}}-C_{j}^{1}$ & 15 & 0 & 0 & 1 & 0 & 0 & 0 & 0 & 0 & 0 & 0 & -3 \\
$Z_{j}^{C^{2}}-C_{j}^{2}$ & 15 & 10
\end{tabular}




\section{Conclusion And COMments}

In this paper, we have proposed an algorithm that can be regarded as a modified and corrected version of Gupta and Malhotra's procedure. The test that they proposed in the terminal step $\left(\Gamma=\emptyset\right.$ and $z_{1 j}-c_{j}^{1}>0$ or $\Gamma_{n} \neq \emptyset$ and for all $j \in \Gamma_{n}$ yields dominated edge) can be held and not yet all the efficient solutions are detected. As an alternative, the $\Gamma_{n}$ set is modified and the cuts of type $\sum_{j \in N_{k} \backslash\left\{j_{k}\right\}} X_{j} \geq 1$ are carried out until obtaining an infeasible solution outside the feasible region $F$.

In addition, solving the problem $(P)$ is not an obvious extension from a continuous case (without integer constrained) and the fundamental principal the so called Geoffrion's theorem is no longer valid in presence of discrete variables, which makes this class of problems very difficult as was stated by Teghem Jr. [17].

Thus, using metods introduced for continous variables, such Ecker's and Kouada can be missleading when applied to problems in the presence of discrete variables.

Acknowledgements. The authors would like to thank Professor J. Abadie for the many helpful suggestions.

\section{REFERENCES}

[1] M. Abbas and M. Moulaï, Solving Multiple Objective Integer Linear Programming Problem. Ricerca Operativa 29 (1999) 15-39.

[2] P. Armand and C. Malivert, Determination of the Efficient Set in Multi-Objective Linear Programming. J. Optim. Theory Appl. 70 (1991) 467-489.

[3] P. Armand, Finding all maximal efficient faces in multi-Objective linear programming. Math. Programming 61 (1993) 357-375.

[4] M.S. Bazaraa and C.M. Shetty, Non linear Programming theory and Algorithms. J. Wiley, New York (1979).

[5] H.P. Benson, Finding an initial Efficient Extreme Point for a Linear Multiple Objective Program. J. Oper. Res. Soc. (1981) 495-498.

[6] H.P. Benson, Existence of Efficient solutions for vector Maximization Problems. J. Optim. Theory Appl. 26 (1978) 569-580.

[7] G.R. Bitran, Linear Multiple Objective Programs with zero-one variables. Math. Programming 13 (1977) 121-139.

[8] J.G. Ecker and I.A. Kouada, Finding Efficient Points for Multi-Objective Linear Programs. Math. Programming 8 (1975) 375-377.

[9] J.G. Ecker and I.A. Kouada, Finding All Efficient Extreme Points for Multi-Objective Linear Programs. Math. Programming 14 (1978) 249-261.

[10] R. Gupta and R. Malhotra, Multi-Criteria Integer Linear Programming Problem. Cahiers Centre Études Rech. Opér. 34 (1992) 51-68.

[11] A.T. Hamdy, Integer Programming, Theory, Applications and Computations. Academic Press (1975).

[12] H. Isermann, The Enumeration of the set of all Efficient solutions for a Linear Multiple Objective Program. Oper. Res. Quarterly 28/3 (1977) 711-725.

[13] D. Klein and E. Hannan, An Algorithm for the Multiple Objective Integer Linear Programming Problem. Eur. J. Oper. Res. 9 (1982) 378-385. 
[14] J. Philip, Algorithms for the Vector Maximization Problem. Math. Programming 2 (1972) 207-229.

[15] B. Roy, Problems and methods with Multiple Objective functions. Math. Programming 2 (1972) 207-229.

[16] R.E. Steuer, Multiple Criteria Optimization theory, Computation and Applications. Wiley, New York (1985).

[17] J. Teghem and P.L. Kunsh, A Survey of Techniques for Finding Efficient Solutions. AsiaPacific J. Oper. Res. 3 (1986) 95-108.

[18] E.L. Ulungu and J. Teghem, Multi-Objective Combinatorial Optimization Problem: A Survey. J. Multi-Criteria Decision Anal. 3 (1994) 83-104.

[19] V. Verma, Constrained Integer Linear Fractional Programming Problem. Optimization 21 (1990) 749-757.

[20] P.L. Yu, Multiple Criteria Decision Making. Plenum, New York (1985).

[21] M. Zeleny and P.L. Yu, The set of all non-dominated solutions in linear cases and Multicriteria simplex method. J. Math. Anal. Appl. 49 (1975) 430-468.

[22] S. Zionts, Integer Programming with Multiple Objectives. Ann. Discrete Math. 1 (1977) 551-562.

To access this journal online: www.edpsciences.org 